\title{
EDITORIAL
}

\section{Interdisciplinarity and multidisciplinarity in research}

The revised Scope of the Journal of the National Science Foundation of Sri Lanka states that the Journal publishes the results of research in all aspects of Science and Technology and that it is open for the publication of Research Articles, Reviews, Research Communications, and Correspondence. In this revision, the number of categories has been reduced from eleven (11) to four (04).

This change was decided upon due to the majority of submissions by the scientists being in the category of Research Articles in the year 2014, which was $80 \%$ of the published submissions. The next highest number of publications, which amounted to about $17 \%$ was in the category of Short Communications. The balance, which was a little over $2 \%$, was in the category of Research Notes. There were no publications in any of the other categories. The high number of submissions in the category of Research Articles is a satisfactory situation. The majority of these research articles report the results of studies conducted in specific disciplines of science. The results of these publications add to the knowledge base of various disciplines. This, no doubt is one of the important responsibilities of researchers in science.

However, in selecting a research study in science, research scientists must keep in mind that their research findings should also contribute to the force that drives innovations, and find answers to pressing problems faced by the society. It is through the research findings that solutions can be worked out for complex problems in such areas as health, food security, disaster management and environment. This points to the necessity and importance of research on studies targeted towards problem solving. Such studies will also generate new knowledge necessary for today's knowledge economy and also find ways and means to solve some problematic issues faced by the society.

The problems faced by our society today such as the chronic kidney disease of unknown etiology ( $\mathrm{CKDu})$ and climate change are extremely complex and cannot be fitted directly into any given disciplinary framework. Impacts of many such problems are felt by the society at large even threatening life as well as their livelihoods. Such problems need investigations and inputs not only from the hard sciences, but also from social sciences. Hence, involvement of social scientists in such research studies is important in today's context. Therefore, it is necessary for the researchers in related fields to work in closely knit teams to find sustainable and innovative solutions. These teams could be either interdisciplinary or multidisciplinary depending on the study to be undertaken.

In interdisciplinary research studies, scientists gather information and data using techniques, tools, perspectives, concepts, and/or theories in two or more disciplines, which are integrated to advance fundamental understanding of an issue or to solve problems whose solutions are beyond the scope of a single discipline. Besiden, in multidisciplinary research, knowledge of different disciplines are gathered by researchers working in their respective disciplines and collate the findings to solve problems.

If the problem being investigated calls for approaches from different angles or disciplines, the researchers will have to develop their study as a multidisciplinary one where the results will have to be combined with an additive approach to find the solutions. If the problem requires an integrated approach to synthesise the perspectives of multiple individual disciplines of study during all phases, it calls for an interdisciplinary study. Across the world, barriers to research studies whether it is disciplinary or institutional can be overcome by taking an interdisciplinary approach.

To develop an interdisciplinary approach the research scientists must cultivate the ability to operate across boundaries of disciplines while keeping in mind the principles of the different disciplines. These researchers must also be able to appreciate the implications of the 
available information in a broader context, and most of all trust and believe in each other with mutual respect and also believe that no one discipline is superior to another. It is seen that increasing specialisation and the resulting compartmentalisation of knowledge results in scientists that prefer to undertake studies in their own area of expertise in a specific discipline. This must be changed to produce a new generation of scientists who will be more responsible to the needs of the society through research, which is either interdisciplinary or multidisciplinary.
The members of this new generation must be able to establish connections among discrete disciplines. It is no doubt a tall order for research scientists to have a broad knowledge across several disciplines with in-depth knowledge of one or two.

It is expected that interdisciplinarity and multidisciplinarity in research will result in innovations and solutions to problems, which are beyond the scope or areas of research of single disciplines.

Nalini Ratnasiri 